\title{
Reproducibility of volumetric measurements of normal maculae with the Heidelberg retina tomograph
}

\author{
H J Zambarakji, J E Evans, W M K Amoaku, S A Vernon
}

\begin{abstract}
Aims-The Heidelberg retina tomograph (HRT) is a scanning laser ophthalmoscope with confocal optics. The reproducibility of the optic nerve head topography is accurate and reliable. The authors describe a new technique for the assessment of macular thickening by volumetric quantification and present the results of its reproducibility in normal subjects. Methods-Topographic images of the macula, centred on the fovea were obtained in one eye of 44 normal subjects. The volumes above the reference plane bound by a $1 \mathrm{~mm}, 2 \mathrm{~mm}$, and $3 \mathrm{~mm}$ diameter circle were measured. The reference plane was adjusted to the lowest point of the height variation of the contour line at each examination. The reproducibility of repeated measurements within a $2 \mathrm{~mm}$ diameter circle was assessed in 20 eyes selected at random. Three HRT scans of each eye were obtained. The measurements of volume above reference plane of each scan were repeated three times on three separate days.
\end{abstract}

Results-The intrascan coefficients of variability measured $7.12-9.57 \%$. The $95 \%$ confidence interval for the geometric mean ratio of single volume measurements was 0.92 to 1.24 for scans 1 and 2, 0.89 to 1.17 for scans 1 and 3 , and 0.81 to 1.12 for scans 2 and 3 . When the mean of three measurements of one scan were compared with the mean of three measurements of a second scan, the $95 \%$ confidence interval for their geometric mean ratio was 0.89 to 1.20 for scans 1 and $2,0.89$ to 1.16 for scans 1 and 3 , and 0.84 to 1.13 for scans 2 and 3 . The average standard deviation (SD) for one measurement per scan was $0.02 \mathrm{~mm}^{3}$, and $0.019 \mathrm{~mm}^{3}$ for two or three measurements per scan. Linear regression demonstrated a significant increase in SD as volumetric measurements increased $(p=0.003)$. Age did not significantly affect the SD of volumetric measurements $(p=0.797)$. The authors found no significant differences in volumetric measurements across all ages for all three circles $(p=0.314, p=0.471, p=0.267)$.

Conclusion-Good reproducibility for volumetric measurements at the macula was found with the HRT using the above technique in normal subjects. This method may be extremely useful for the identification and quantification of dia- betic macular oedema and for monitoring the effects of argon laser photocoagulation.

(Br f Ophthalmol 1998;82:884-891)

Traditional methods of evaluating the macular area including slit lamp biomicroscopy and stereo fundus photography are relatively insensitive to small changes in retinal thickness. ${ }^{1}$ Fluorescein angiography has unavoidable side effects ${ }^{2}{ }^{3}$ such as yellowing of the skin and urine but more serious side effects range from the less severe urticaria to the life threatening anaphylactic reactions. Current methods for in vivo assessment of macular pathology such as diabetic macular oedema include digitised fundus fluorescein angiography ${ }^{45}$ and optical coherence tomography. ${ }^{6}$

Confocal scanning laser tomography was originally designed for the analysis of optic nerve head topography ${ }^{7}$ and has more recently been adapted for the investigation of the macula. ${ }^{89}$ Good reproducibility for point height and mean depth measurements at the macula were recently demonstrated with the Heidelberg retina tomograph (HRT).$^{10}$ Furthermore, the analysis of retinal reflectivity as a function of scan depth ( $Z$ profile) with the HRT has recently been described to derive topographic macular oedema $\operatorname{maps}^{11}$ as a measure of retinal thickening in three selected patients and 14 normal subjects.

The HRT allows safe and reproducible measurements of the optic nerve head. One study ${ }^{12}$ assessed the effect of repetitive imaging on topographic measurements of the optic nerve head with the HRT. The reproducibility of the instrument as measured by average standard deviation (SD) was $35.5 \mu \mathrm{m}$ for one examination per visit; this improved to $25.7 \mu \mathrm{m}$ for three examinations per visit. Another study ${ }^{13}$ found a test-retest variability of topographic measurements of optic nerve head and parapapillary retina of $31.20 \mu \mathrm{m}$ and $25.94 \mu \mathrm{m}$ in glaucoma patients and controls respectively $(\mathrm{p}=0.01)$ as well as a significant increase in variability of topographic measurements with age $(p<0.001)$. The coefficients of variation for optic cup volumes in a recent study ranged from $1 \%$ to $16.4 \%$ in normal subjects and 1.3 to $13 \%$ in glaucoma subjects. ${ }^{14}$

It is important to know whether the reproducibility of optic nerve cup and rim volume applies to the topographic measurements at the macula. Although the topography of the macula has been investigated by other authors, ${ }^{89}$ there is a distinct lack of data on the 


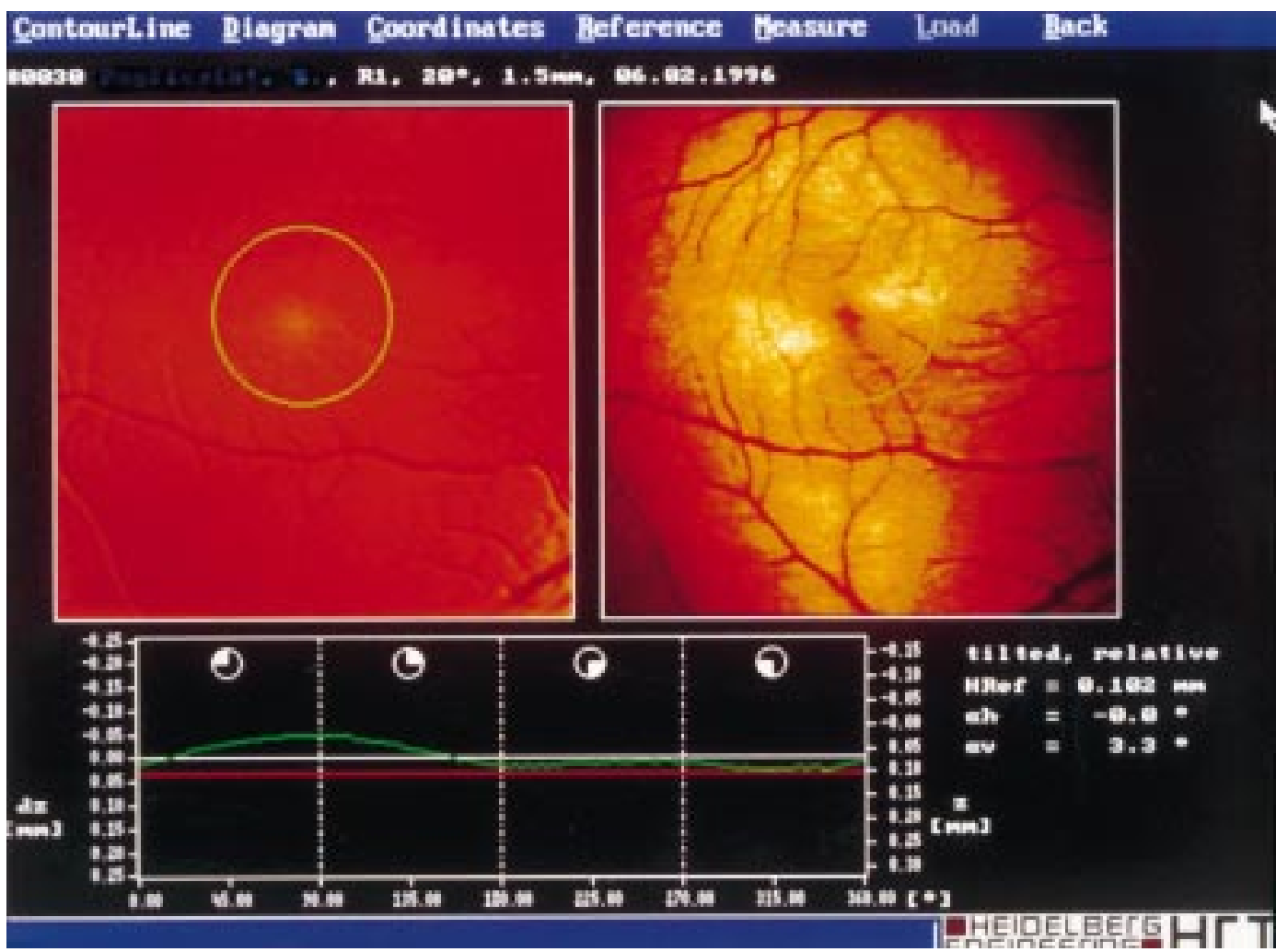

Figure 1 HRT scan of a normal macula in a healthy 36 year old man. The height variation of the contour line is shown in green and the reference plane (in red) is adjusted to the lowest point of the contour line. The scan is centred at the fovea. The circle centre is the fovea, the diameter is $2 \mathrm{~mm}$. The scale $(25 \mathrm{~mm})$ is given on the left hand vertical axis.

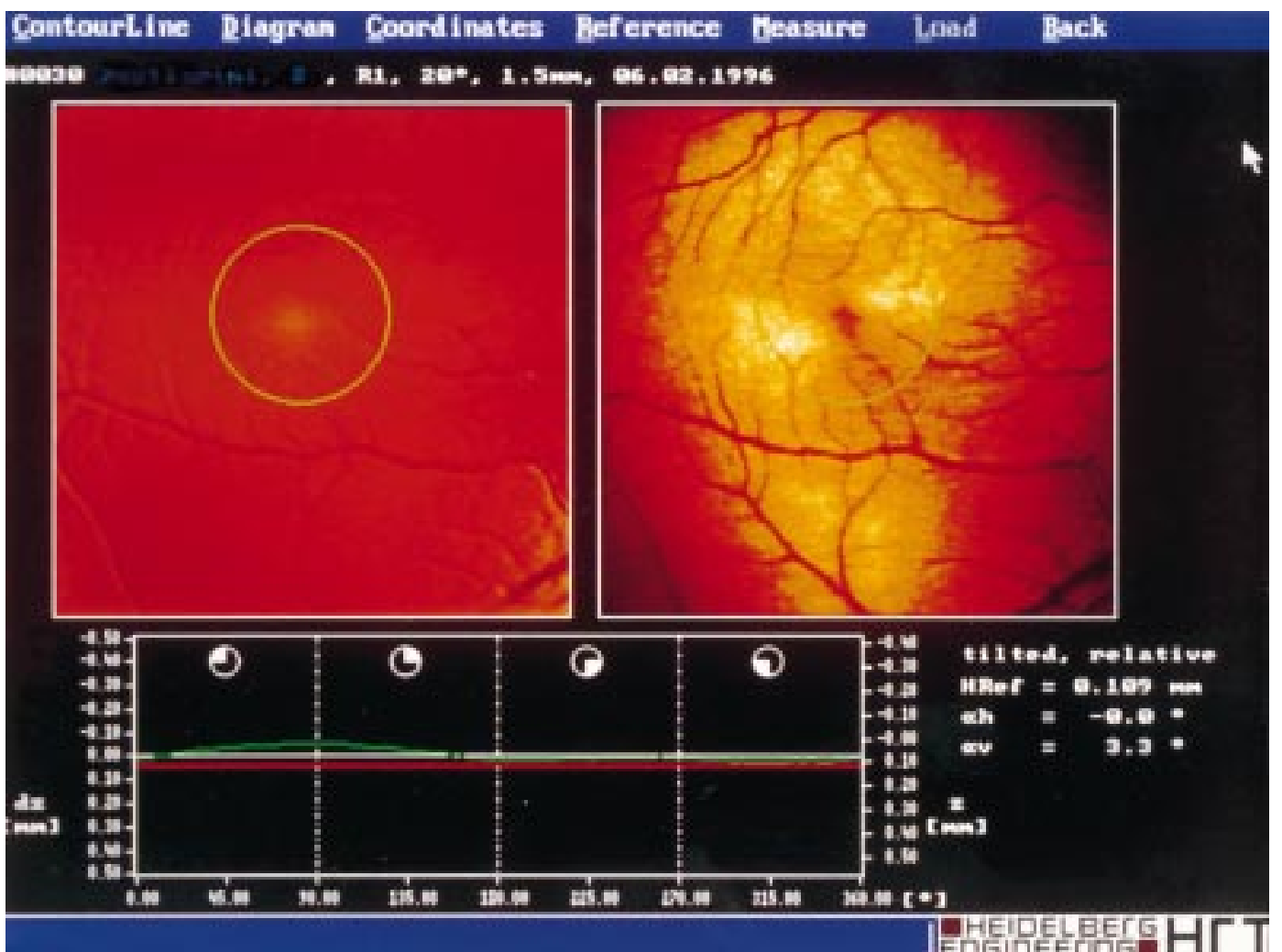

Figure 2 HRT scan of the same macula given in Figure 1. The scale is adjusted to $50 \mathrm{~mm}$, the contour line is almost a perfect straight line.

reproducibility of such measurements. We have previously described a new method for the assessment of the macula by volumetric analysis with the HRT using the software version 1.11 provided by Heidelberg. ${ }^{15}$ The present study is an analysis of the reproducibility of this measurement technique in 20 normal macu- lae, and a description of the variation of measured volumes across the age range in 44 control eyes.

\section{Patients and methods}

The HRT is a scanning laser ophthalmoscope (SLO) with confocal optics. ${ }^{9}$ Technical details 
of the instrument have been described elsewhere. ${ }^{12}{ }^{13}$ The scanning head of the HRT was mounted to a standard ophthalmic stand with a head rest. The patient's retinal surface was focused on the monitor screen by adjusting the location of the focal plane, the best focus being directly related to subject's refractive error. The $670 \mathrm{~nm}$ wavelength diode laser was used to image the macula using a $20^{\circ}$ by $20^{\circ}$ field of view. The total scan depth was adjusted according to the thickness of the structure to be analysed from 0.50 to $4.00 \mathrm{~mm}$. When a normal retina is imaged (Figs 1 and 2), a scan depth of $0.50-1.50 \mathrm{~mm}$ is usually required for a good quality scan; however, eyes with macular thickening require a total scan depth of up to $4.0 \mathrm{~mm}$. In this study the initial scan depth was set at $1.00 \mathrm{~mm}$ before the images were taken. If an adjustment was required the HRT software automatically displayed a signal and the scan depth was adjusted accordingly.

The instrument takes 32 images in 1.6 seconds in a plane perpendicular to the optical axis. The confocal system detects light reflected from the focal plane and suppresses reflections from axial locations outside the focal plane. The resulting topographic map displays the height of the retinal surface from the focal plane of the eye. The computer software provided by Heidelberg aligns the 32 cuts so that all eye movements are removed and then compiles the images into a three dimensional image.

\section{HRT IMAGE ACQUISITION AND TECHNIQUE} DESCRIPTION

All examinations were performed after cycloplegia using cyclopentolate $1 \%$ to minimise the variability of measurements. ${ }^{10}$ The images were centred on the fovea and a circle was drawn using the circle draw facility. The circle centre was the fovea, the cursor was positioned at the foveal centre as seen on the monitor screen and then moved radially to draw a circle. The circle radius was then adjusted using the HRT software to the size required and the final image stored. The circle could be moved to the side and recentred to ensure the fovea was at its centre. The height of any point on the circle is given by the contour line in green (Fig 1). The reference plane shown in red (Fig 1) was adjusted to the lowest point of the contour line and the volume above reference plane was calculated by the computer software.

STUDY EYES

In all, 44 eyes of 44 normal subjects were included in the study. All subjects underwent a complete ophthalmic examination, including visual acuity testing on a Snellen chart, slit lamp biomicroscopy, and dilated stereoscopic ophthalmoscopy with a yellow coated 78 dioptre (D) Volk lens by one experienced examiner (HJZ). Those with coexistent past or present ocular disease were excluded from the study. HRT scans were obtained by one experienced SLO operator (HJZ). All eyes were within $5.50 \mathrm{D}$ of emmetropia (best sphere range -5.50 to $+4.75 \mathrm{D})$ with a maximum cylindrical correction of plus or minus $1.00 \mathrm{D}$.
Mean age was 45.93 years (range 22-76). All subjects had $6 / 5$ corrected visual acuity.

Twenty eyes of 20 subjects were selected at random for the reproducibility analysis of this technique. All were within $5.50 \mathrm{D}$ of emmetropia (range -5.50 to +4.00 ). Mean age was 49.30 years (range $25-76$ ).

Informed consent was obtained for each subject which adhered to the tenets of the Declaration of Helsinki. The study protocol had ethics committee approval by the review board of the Queen's Medical Centre of the University Hospital of Nottingham.

VOLUME ABOVE REFERENCE PLANE $V$ AGE

The volumes above reference plane of three circles measuring 1,2 , and $3 \mathrm{~mm}$ in diameter respectively were measured on three different days by the same observer (HJZ) on all 44 eyes. The circle was erased at the end of each examination so that its centre (the fovea) and the reference plane had to be redefined every time. Regression equations and correlation coefficients were calculated using the mean of three measurements for each circle size.

\section{REPRODUCIBILITY}

Three scans of each of 20 eyes were obtained by the method described. The subjects were asked to sit back between each scan and the head position was readjusted in order to simulate a separate examination. We selectively accepted scans with good acquisition variables - that is, clear, well illuminated scans centred on the fovea. One experienced SLO operator $(\mathrm{HJZ})$ then measured the volume above reference plane within a $2 \mathrm{~mm}$ diameter circle by the method described above. To examine the repeatability of measurements, each circle was redrawn three times on each of three different days for every scan. The circles were erased after every examination so that the centre (the fovea) and the reference plane had to be redefined every time.

\section{STATISTICAL ANALYSIS}

Intraobserver variability was assessed by regression equations and by calculation of coefficients of variability. Coefficients of variability were calculated as the square root of the mean value of the variance of the measurements divided by the mean measured volume above reference plane.

Agreement between measurements were measured by techniques described by Bland and Altman. ${ }^{16}$ As the differences were related to the mean over the range of measurements, a logarithmic transformation of the data was performed. ${ }^{16}$ Bias between the mean of three

Table 1 Mean volume above reference plane $\left(\mathrm{mm}^{3}\right)$ in a group of 44 normal eyes for the $1 \mathrm{~mm}, 2 \mathrm{~mm}$, and $3 \mathrm{~mm}$ diameter circles. The standard deviation of measurements are also given

\begin{tabular}{lll}
\hline & $\begin{array}{l}\text { Mean volume } \\
\left(\mathrm{mm}^{3}\right)\end{array}$ & $\begin{array}{l}\text { Standard } \\
\text { deviation } \\
\left(\mathrm{mm}^{3}\right)\end{array}$ \\
\hline $1 \mathrm{~mm}$ diameter circle & 0.016 & 0.006 \\
$2 \mathrm{~mm}$ diameter circle & 0.1 & 0.035 \\
$3 \mathrm{~mm}$ diameter circle & 0.369 & 0.151 \\
\hline
\end{tabular}




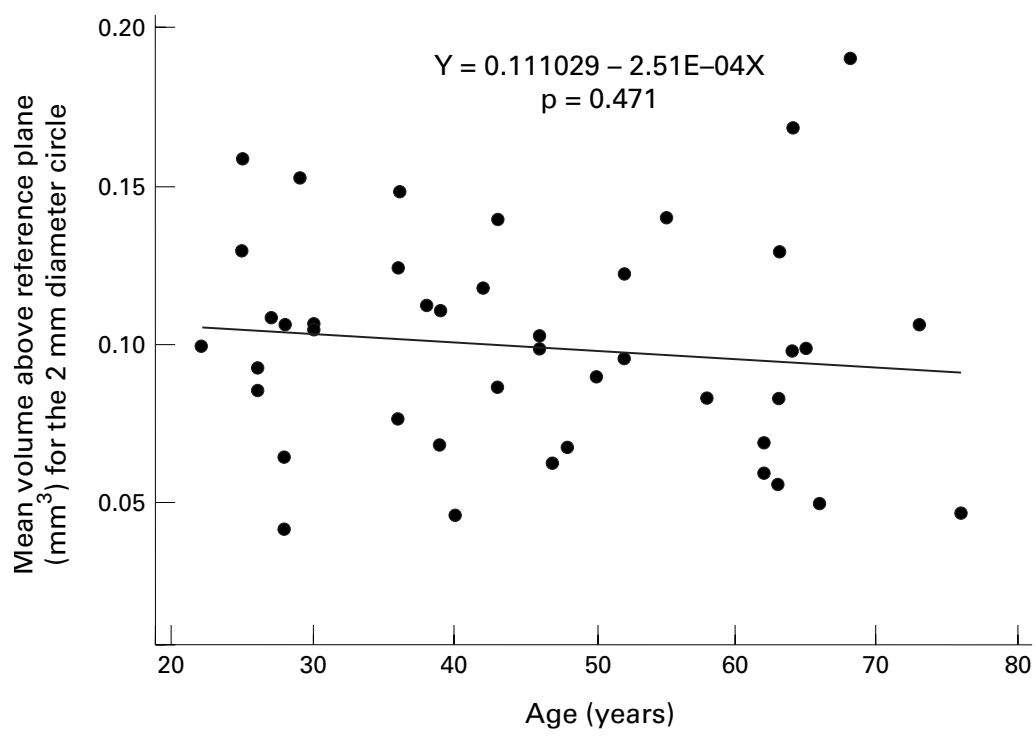

Figure 3 Regression line of the mean volume above reference plane of three examinations of a $2 \mathrm{~mm}$ diameter circle $v$ age. There is poor correlation between the volumetric measurements and age $(r=-0.112, p>0.1)$. The slope of the regression line is not significant $(p=0.471)$ indicating no significant variability in volumes over the age range.

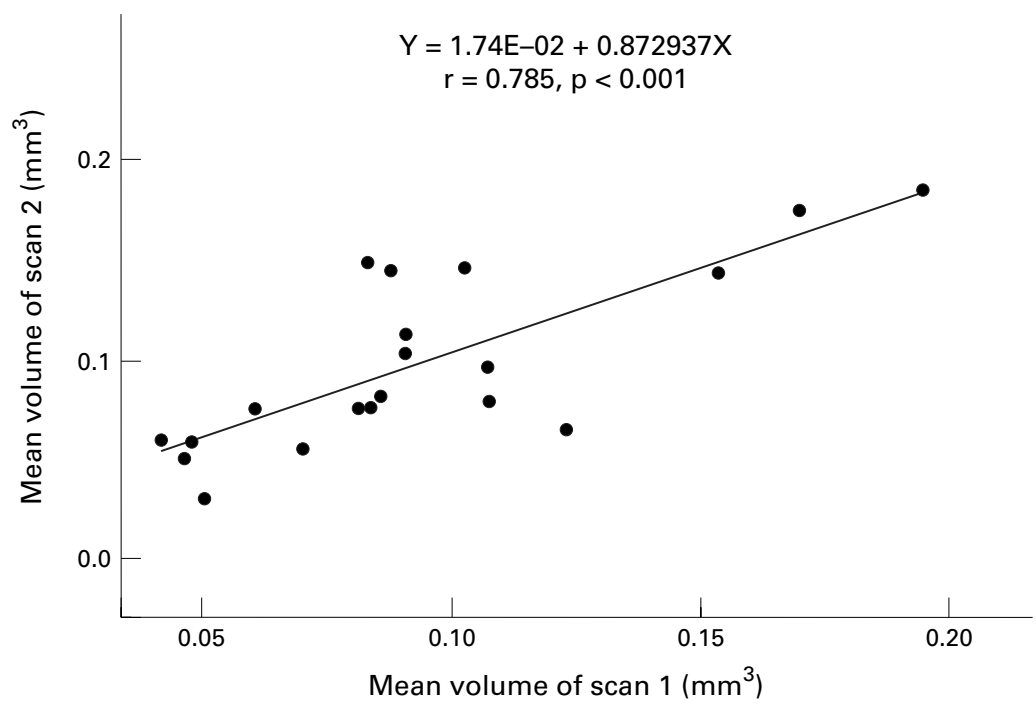

Figure 4 There is good correlation between the mean volume of three examinations of scan $1 \mathrm{v}$ the mean volume of three examinations of scan 2 within the $2 \mathrm{~mm}$ diameter circle $(r=$ $0.785, p<0.001)$. $\log$ counts. The antilog of the difference in sample means on the log transformed scale is by definition, an estimate of the ratio of the two population geometric means, and the antilogged CI for the difference gives a CI for this ratio. ${ }^{17}$ As the $\mathrm{CI}$ for the difference between log counts is not as easy to interpret as a CI relating to the actual counts, we can antilog the values describing the CI for the difference between log counts to obtain a $95 \%$ CI for the geometric mean ratio of the actual volumetric measurements. This calculation makes use of the fact that differences between the logarithm of two quantities is exactly the same as the logarithm of their ratio.

Thus, $\log ($ mean scan 1$)-\log ($ mean scan 2$)$ $=\log ($ mean scan $1 /$ mean scan 2$)$.

Linear regression analysis was performed to determine the relation of the SD of nine examinations of each eye and age as well as the relation of SD and volumetric measurements. As other authors have used the average SD as a measure of reproducibility, we have calculated the average SD of one examination per scan and performed similar calculations for two and three examinations per scan.

Findings with an error probability value of less than 0.05 were considered statistically significant.

\section{Results}

VOLUME ABOVE REFERENCE PLANE $V$ AGE

We found no significant differences in volumetric measurements for all three circle sizes ( $1 \mathrm{~mm}, 2 \mathrm{~mm}$, and $3 \mathrm{~mm}$ diameter) across all age groups. The slope of the regression lines of volumes above reference plane against age was not significantly different from zero $(\mathrm{p}=0.314$, $p=0.471, p=0.267$, Fig 3 ). There was no significant correlation between volume and age $(r$ $=0.155, r=-0.112, r=-0.171$ for the $1 \mathrm{~mm}$, $2 \mathrm{~mm}$, and $3 \mathrm{~mm}$ diameter circles respectively, $\mathrm{p}>0.1)$. The mean volumetric measurements and standard deviations are summarised in Table 1.

\section{REPRODUCIBILITY}

There was good correlation between the mean of three examinations of each scan $(\mathrm{p}<0.001$, Fig 4, Table 2). Agreement between scans was examined by plotting the difference between the $\log$ values of the mean of three examinations against the mean of the log values. ${ }^{16} \mathrm{We}$ found good agreement as the mean differences of the logs were 0.016 for scans 1 and 2 (Fig 5), 0.008 for scans 1 and 3, and -0.008 for scans 2 and 3. Neither scan tended to read higher or lower than the other two as the slope of the regression lines were not significant $(p=0.428$, $\mathrm{p}=0.503, \mathrm{p}=0.168$ respectively).

The intrascan coefficients of variation were $8.26 \%$ for scan $1,7.12 \%$ for scan 2 , and $9.57 \%$ for scan 3 . However the interscan coefficient of variation was $20.14 \%$.

The $95 \%$ CI of the geometric mean ratio of one examination of scan 1 compared with one examination of scan 2 was 0.92 to 1.24 . When we compared the mean of three examinations of scan 1 with the mean of three examinations of scan 2, the $95 \%$ CI of their ratio was 0.89 to

\begin{tabular}{lll}
\hline Mean scan $1 v$ mean scan 2 & 0.785 & $<0.001$ \\
Mean scan 1 $v$ mean scan 3 & 0.740 & $<0.001$ \\
Mean scan 2 $v$ mean scan 3 & 0.683 & $<0.001$
\end{tabular}




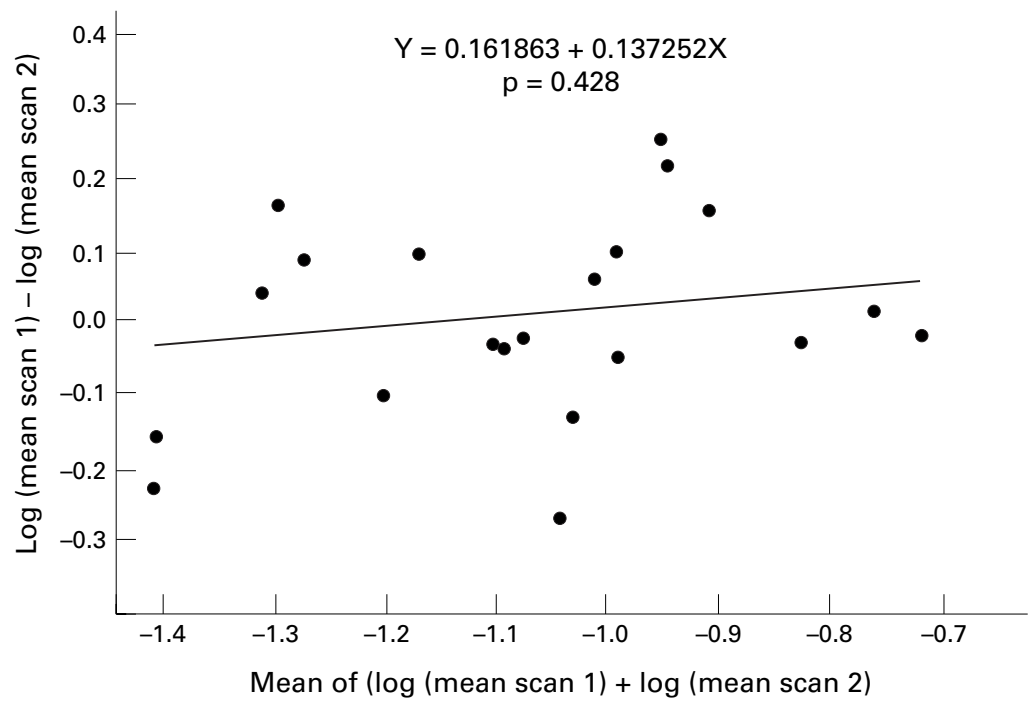

Figure 5 Agreement between scan 1 and scan 2 is examined by plotting the difference between the log values of the mean of three examinations against the mean of the log values. The slope of the regression line is not significant $(p=0.428)$ and the mean difference of the log transformed data (0.016) is almost zero.

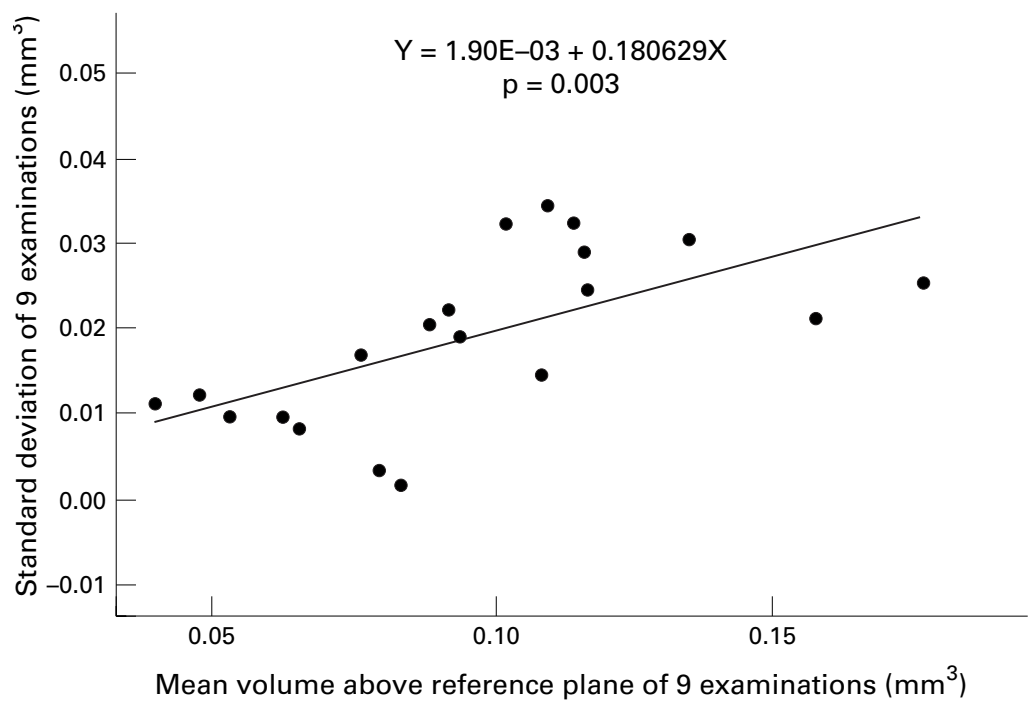

Figure 6 Standard deviation of nine examinations of three scans (three examinations per scan) $v$ mean measured volume. The slope of the regression line is significant $(p=0.003)$ demonstrating an increase in standard deviation with greater volumes.

1.20. These results demonstrate very little difference between the two measured CI, indicating that one careful examination of one good quality scan of a normal macula is as reproducible as the mean of three examina- tions. The results of the $95 \% \mathrm{CI}$ for the other scans are similar to those of scans 1 and 2 and are summarised in Tables 3 and 4 .

The average SD of one examination per scan over the three scans was calculated as a further measure of reproducibility. The same calculations were repeated for the mean of two and three examinations per scan. The SD were $0.020 \mathrm{~mm}^{3}$ for one examination per scan, and $0.019 \mathrm{~mm}^{3}$ for two or three examinations per scan. This is in keeping with the results of the 95\% CI previously calculated.

The SD of all nine measurements of three scans (three examinations per scan) were compared with the mean measured volume above reference plane. The slope of the regression line was significant $(p=0.003)$ demonstrating an increase in SD as the absolute volumetric measurements increased (Fig 6). We found a significant correlation between SD and volumes measured $(r=0.637, \mathrm{p}<0.01)$. However, linear regression analysis showed no significant trend in the SD throughout the age range ( $p=$ 0.797 , Fig 7) and no correlation between SD and age $(r=0.061, \mathrm{p}>0.1)$.

\section{Discussion}

Confocal scanning laser ophthalmoscopy of the macula represents a major advance in the ability to analyse its topography. Our study describes a new technique for the volumetric assessment of the central macula ( $2 \mathrm{~mm}$ diameter circle centred at the fovea). This area was chosen to provide sufficient information about the area most critical to vision. The initial assessment of three different sized circles was to establish whether the variability in volumes across the age range was dependent on the circle size or not. However, the reproducibility was examined for the $2 \mathrm{~mm}$ diameter circle only as this represents an area of particular concern in diabetic patients with maculopathy. Thus, all cases of clinically significant macular oedema would be identified unless the zone of retinal thickening was one disc area in size, at least part of which was within one disc diameter of the centre of the macula, but outside the area covered by the $2 \mathrm{~mm}$ diameter circle. Although some cases might, in theory, not be identified on volumetric measurement, we feel that the three dimensional map images

Table 3 One examination of one scan compared with one examination of another scan. The 95\% CI for the mean difference of log transformed values were initially calculated and the antilogs are quoted as the $95 \%$ CI of the geometric mean ratio of the volumes of scans 1 and 2,1 and 3 , and 2 and 3

\begin{tabular}{lccc}
\hline & Scan 1 v scan 2 & Scan 1 v scan 3 & Scan 2 v scan 3 \\
\hline Mean difference of log values & 0.0293 & 0.0107 & -0.0187 \\
Standard error of the differences of log values & 0.0309 & 0.0290 & 0.0335 \\
$95 \%$ CI for the mean difference of log values & -0.0354 to 0.094 & -0.05 to 0.0713 & -0.0887 to 0.0514 \\
$95 \%$ CI for the geometric mean scan ratio & 0.92 to 1.24 & 0.89 to 1.17 & 0.81 to 1.12 \\
\hline
\end{tabular}

Table 4 Mean of three examinations of one scan compared with the mean of three examinations of another scan. The 95\% CI for the mean difference of log transformed values were initially calculated and the antilogs are quoted as the 95\% CI of the geometric mean ratio of the volumes of scans 1 and 2, 1 and 3, and 2 and 3

\begin{tabular}{lccc}
\hline & Scan 1 v scan 2 & Scan 1 v scan 3 & Scan 2 vsan 3 \\
\hline Mean difference of log values & 0.0168 & 0.0086 & -0.0082 \\
Standard error of the differences of log values & 0.0302 & 0.0267 & 0.0311 \\
$95 \%$ CI for the mean difference of log values & -0.0463 to 0.08 & -0.0474 to 0.0646 & -0.0734 to 0.0569 \\
$95 \%$ CI for the geometric mean scan ratio & 0.89 to 1.20 & 0.89 to 1.16 & 0.84 to 1.13 \\
\hline
\end{tabular}




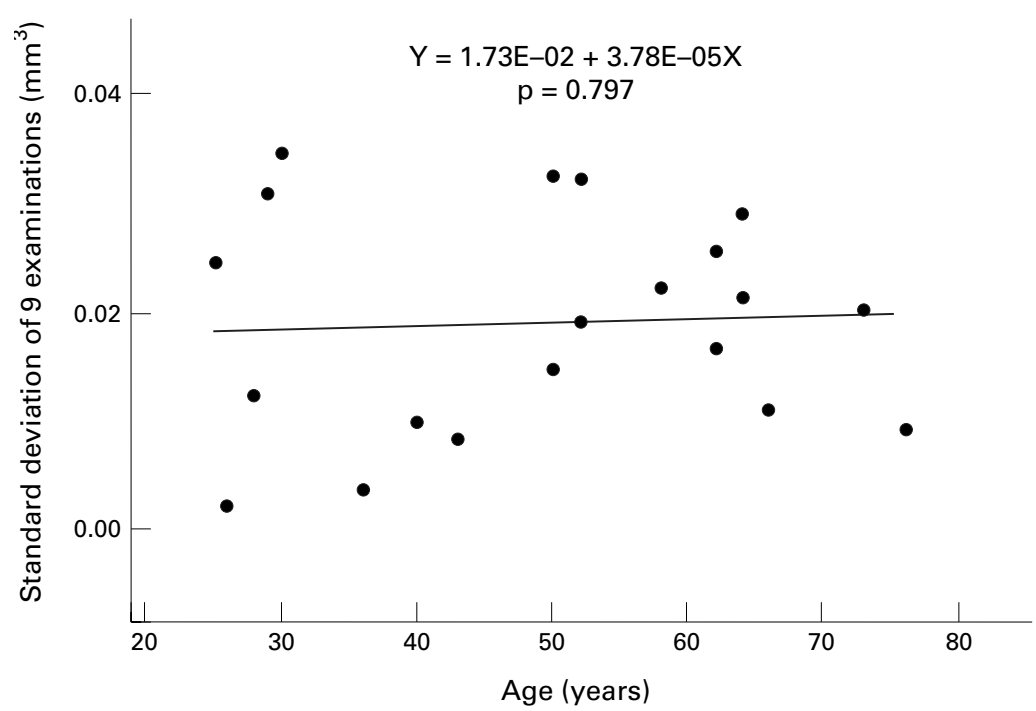

Figure 7 The regression plot of standard deviation of nine examinations of three scans (three examinations per scan) $v$ age demonstrates no significant variability across the age range $(p=0.797)$, and no correlation between standard deviation and age $(r=0.061, p>$ $0.1)$.

of the software would identify those not detected on volume measurement alone by producing a retinal elevation map of the central $20^{\circ}$ of the posterior pole.

The anatomical fovea (or clinical macula) is the $1.5 \mathrm{~mm}$ diameter central retina centred at the foveola (0.35 $\mathrm{mm}$ diameter depression). The shallowly sloping peripheral fovea starts $750 \mu \mathrm{m}$ from the centre, the retina being thinnest in the foveal pit $(100 \mu \mathrm{m})$ and thickest in the perifoveal region $(230 \mu \mathrm{m}){ }^{18}$ The volume measured is therefore the volume of the superior part of a doughnut shaped structure whose outer diameter measures $2 \mathrm{~mm}$. The volumes measured in controls are small thus reflecting the shape of the normal retina. The volumetric measurements in diabetic macular oedema are statistically greater than controls, ${ }^{15}$ indicating that the volume measured in diabetic maculopathy is that of a normal macula plus an additional volume attributable to retinal thickening. The height contour of the 2 $\mathrm{mm}$ diameter circle centred at the foveola (or clinical fovea) should theoretically be a straight line (Fig 2). On HRT, the scaling can be adjusted as an "apparent" straight contour line when using the $0.50 \mathrm{~mm}$ scale (Fig 2) may demonstrate some irregularities on the 0.25 $\mathrm{mm}$ scale (Fig 1). Any variation in the contour line should not therefore be considered as true elevation but as a relative elevation. It is particularly important to centre the scans and the circles drawn at the fovea and to be very accurate with the positioning of the reference plane. The latter can be achieved by moving the scaling to $0.25 \mathrm{~mm}$ which would magnify any variations of the contour line.

The reproducibility results of our paper are presented in a number of ways in order to enable the readers to compare this work with other published data. However, the results of the $95 \%$ CI calculations are the easiest to interpret as they would enable us to determine whether a change in volumetric measurement over time of the same macula represents a true change or is due to expected variability alone.
The reproducibility of repeated topographic images of the optic nerve head and peripapillary retina substantially increase from one to two examinations. ${ }^{12}$ However, with three or more examinations, only slight improvement (SD of $25.7 \mu \mathrm{m}$ with three examinations and $22.5 \mu \mathrm{m}$ with five examinations) was observed indicating that a series of three examinations provides an acceptable balance between the number of examinations per scan and reproducibility. In our study, however, the average SD did not vary with the number of examinations indicating that one examination per scan is as reproducible as the mean of three examinations per scan. This is true for a normal macula, but may not be applicable in cases of diabetic maculopathy.

The ratio of the volumes of two scans of the same eye is given by the $95 \%$ CI (Tables 3 and 4) and confirms that only minimal improvement in reproducibility is observed in our study with the mean of three examinations per scan compared with one examination per scan. Our results (Table 4, scan $1 v$ scan 2) indicate that if scan 1 measured $0.1 \mathrm{~mm}^{3}$, a second scan (scan 2) of the same eye will measure between $0.080 \mathrm{~mm}^{3} \quad(0.1: 1.24)$ and $0.108 \mathrm{~mm}^{3}$ $(0.1: 0.92)$. Similarly, if the mean of three examinations of scan 1 measure $0.1 \mathrm{~mm}^{3}$, the mean of three examinations of another scan (scan 2) will measure between $0.083 \mathrm{~mm}^{3}$ $(0.1: 1.20)$ and $0.112 \mathrm{~mm}^{3}(0.1: 0.89)$.

The coefficient of variability is frequently quoted in reproducibility studies. This was $7.12 \%$ to $9.54 \%$ in our study (within scan), similar to figures quoted for normal optic nerve cup volumes (mean $4.6 \%$, range $1 \%$ to $16.4 \%) .{ }^{14}$ This increased on interscan variability to $20 \%$ in our study, despite our attempt to minimise measurement error by cycloplegia and particular attention to the positioning of the circle centre and the reference plane. Both within and between scan variability are dependent on similar elements-that is, centring the circle accurately and positioning the reference plane, as every measurement of each scan is performed without any indication of the previous circle's centre and reference plane locations. Therefore, it is the minimal depth separation that can be detected between two points by the HRT - that is, its depth resolution, which accounts for the differences between the within and between scan variations.

Although the effect of interobserver variability has not been analysed in this study, the authors would expect this to be very similar to the within scan variability as it is our experience that two different observers agree almost perfectly when positioning the circle at the fovea and placing the reference plane at the lowest point of the height variation of the contour line in a normal macula. This is not necessarily the case in diabetic maculae and is currently the subject of a separate study.

However, the volumes measured in diabetic macular oedema are greater than control volumes by an average factor of $2.45(245 \%),{ }^{15}$ well over the $20 \%$ variability which may occur in controls. When such "large" volumes are measured, a greater measurement error can be 
allowed if the resulting volume differs significantly from the mean volume of a group of controls.

Furthermore, the reproducibility of mapping retinal thickness at the posterior pole has an average variability of $4.1 \%$ in normal maculae, ${ }^{19}$ although previous work showed an increased variability of up to $23 \%$ for measurements at the foveola indicating the sensitivity of the technique to minimal displacement. ${ }^{20} \mathrm{As}$ the retinal thickness analysis technique uses a composite of nine scans to produce a $20^{\circ}$ map of the central region of the posterior pole, we would anticipate some overlapping of the nine separate scans which would therefore require manual rearrangement. The technique, however, has excellent depth resolution and precision and is thus likely to have promising applications in the management of diabetic macular oedema. However, one potential disadvantage of the retinal thickness analysis is the need to obtain data from every point or pixel imaged, whereas the measurement of volumes with the HRT yields one measurement only for a circle of predetermined size making the latter simpler and faster to apply in a busy clinical setting.

One further study ${ }^{21}$ indicated the importance of misalignment between the subject and the laser scanner. The authors also hypothesised that pupillary dilatation would exacerbate the problem although misalignment also occurs with undilated pupils. This contradicts findings reported by Menezes et $a l^{10}$ who obtained significantly lower pooled SD for mean height measurements for cyclopleged eyes $(36.0 \mu \mathrm{m})$ compared with undilated eyes $(47.4 \mu \mathrm{m})$. The absence of accommodation and improved image quality obtained with cycloplegia seem unlikely to impair significantly measurement error compared with data obtained with undilated pupils. We feel the theoretic worsening of misalignment with pupillary dilatation is largely offset by the improved quality of the scan and benefits of absent accommodation as most of the variability appears attributable to misalignment per se. Ocular movements during scanning (1.6 seconds) are detected by examination of all 32 scans simultaneously as well as being corrected by the HRT software. In our study, scans with any detectable movement were excluded. Others, ${ }^{22}$ however, reported no significant difference in variability of optic cup volume between undilated and dilated cyclopleged eyes, using the laser tomographic scanner, the original version of the HRT. Furthermore, Spencer et $a l^{23}$ reported variability coefficients of less than $2 \%$ for vertical optic disc diameters by HRT. This measurement is for a two dimensional structure which would partly account for the lower coefficients measured. Although narrower CI for mean height and mean depth measurements at the macula are obtained when the average of three examinations are compared, ${ }^{10}$ as opposed to comparing one single examination of two scans, this study $^{10}$ does not give the values for the mean differences or standard error of the differences. It is not clear whether the resulting CI quoted include the mean difference in observations, making these results difficult to interpret. However, the authors correctly raised the issue of measuring the reproducibility of topographic measurements at the macula as this may differ from the reproducibility of optic disc topography.

Our study is a measure of the reproducibility of the volumetric measurement technique at the macula but does not evaluate the accuracy of topographic measurements (neither do other reproducibility studies of HRT macular measurements). One study of the accuracy of topographic measurements with the HRT using a plastic eye model of the human eye to simulate six optic nerve head papillae and several cone shaped elevations reported a pooled relative error of $11 \%$ for the "volume below contour" and "volume above contour" of the optic nerve papillae and $3.8 \%$ for retinal elevations. ${ }^{24}$ The evaluation of the accuracy of the "volume above reference plane" variable at the macula would therefore require a separate study using a model eye because the use of postmortem human eyes is limited by fixation artefacts. This would be particularly relevant to separate normal variation in macular volumes from any inaccuracies of the measurement technique. The authors feel that the large range of volumes $(400 \%$ at worst) observed in this study is probably the result of the normal variation in normal maculas as observer variability was measured at $20 \%$ only on inter scan variability. The anticipated clinical role of this measurement technique would be for the assessment and quantification of diabetic macular oedema, as well as monitoring macular laser treatment and possibly in screening for sight threatening maculopathy.

Our results of SD $v$ volumes are consistent with those of Orgul et $a l^{21}$ who showed that the variability of optic cup volumes was more pronounced with larger measurements.

The SD of volumetric measurements did not vary significantly with age in our study (p $=0.797$, Fig 7), similar to the results of SD of optic cup volumes $v$ age by Rohrschneider et al $(r<0.33, \mathrm{p}>0.2) .{ }^{14}$ However, the variability of height measurements at the optic disc did show a significant but poor correlation with age $(r=0.412, \mathrm{p}=0.0092) .{ }^{14}$ Another study has also demonstrated an increased variability of topographic measurements of the optic nerve head and parapapillary retina with age. ${ }^{13}$ The results of variability of topographic measurements appear to vary to a greater extent in areas of high topographic slopes such as the cup border and blood vessels ${ }^{13}$ where a small alignment error would result in a large disparity in the topography at that particular location. We would therefore expect an increased variability when the reproducibility of our technique is measured in cases of diabetic maculopathy. The measurement of confidence intervals would, however, enable the operator to separate with reasonable confidence normal scans from diabetic macular oedema scans on the basis of volumes above reference plane alone. 
In conclusion, this study establishes the reference values for healthy eyes using a new technique for the volumetric assessment of the macula with the HRT. The reliability of repeated measurements is measured using $95 \%$ confidence intervals. It is likely that the application of this method of analysis will prove particularly useful for the quantification of diabetic macular oedema and the monitoring of macular laser photocoagulation with follow up scans. Further work is currently under way to determine the reproducibility, reference values, and interindividual variation in diabetic maculopathy.

1 Shahidi M, Ogura Y, Blair NP, et al. Retinal thickness analysis for quantitative assessment of diabetic macular oedema. Arch Ophthalmol 1991;109:1115-19.

2 Amalric P, Piau C, Fenies MT. Incidents et accidents au cours de l'angiographie fluoresceinique. Bull Soc Ophtal Fr 1968;68:968-73.

3 Burtner RW, McPherson AR. Adverse reactions in intravenous fluorescein angiography. Ann Ophthalmol 1983;15:1084-6.

4 Arend O, Remky A, Elsner AE, et al. Quantification of cystoid changes in diabetic maculopathy. Invest Ophthalmol Vis Sci 1995;36:608-13.

5 Phillips RP, Ross RG, Tyska M, et al. Detection and quantification of hyperfluorescent leakage by computer analysis of fication of hyperfluorescent leakage by computer analysis of fundus fluorescein angiog

6 Hee MR, Puliafito CA, Wong C, et al. Quantitative assessment of macular oedema with optical coherence tomography. Arch Ophthalmol 1995;113:1019-29.

7 Kruse FE, Burk ROW, Volcker HE, et al. Reproducibility of topographic measurements of the optic nerve head with lase tomographic scanning. Ophthalmology 1989;96:1320-4.

8 Burk ROW, Rohrschneider K, Volcker HE. Macula evaluation by laser scanning tomography. In: Nasemann JE, Burk ROW, eds. Scanning laser ophthalmoscopy and tomography. Munich: Ouintessenz Verlags, 1990:225-36.

9 Bartsch D-U, Intaglietta M, Bille JF, et al. Confocal laser tomographic analysis of the retina in eyes with macular hole formation and other focal macular diseases. $A m ~ f$ Ophthalmol 1989;108:277-87.

10 Menezes AV, Giunta M, Chisholm L, et al. Reproducibility of topographic measurements of the macula with scanning laser ophthalmoscope. Ophthalmology 1995;102 $230-5$
11 Hudson C, Flanagan JG, Turner GS, et al. Scanning laser tomography $\mathrm{Z}$ profile signal width as an objective index of macular retinal thickening. Br f Ophthalmol 1998;82:12130.

12 Weinreb RN, Lusky M, Bartsch D-U, et al. Effect of repetitive imaging on topographic measurements of the optic nerve head. Arch Ophthalmol 1993;111:636-8.

13 Chauhan BC, LeBlanc RP, McCormick TA, et al. Test-retest variability of topographic measurements with confocal scanning laser tomography in patients with glaucoma and control subjects. Am f Ophthalmol 1994;118: 9-15.

14 Rohrschneider K, Burk ROW, Kruse FE, et al. Reproducibility of the optic nerve head topography with a new laser tomographic scanning device. Ophthalmology 1994;101:1044-9.

15 Zambarakji HJ, Amoaku WM, Vernon SA. Volumetric analysis of early macular edema with the Heidelberg retina tomograph in diabetic retinopathy. Ophthalmology (in press).

16 Bland JM, Altman DG. Statistical methods for assessing agreement between two methods of clinical measurement. Lancet 1986;i:307-10.

17 Gardner MJ, Altman DG. In: Statistics with confidenceconfidence intervals and statistical guidelines. London: BMJ Publishing Group, 1989:20-27.

18 Straatsma BR, Foos RY, Spencer LM. In: The retinatopography and clinical correlations. New Orleans Academy of Ophthalmology: Symposium on retina and retinal surgery. St Louis: CV Mosby, 1969:1-26.

19 Zeimer R, Shahidi M, Mori M, et al. A new method for rapid mapping of the retinal thickness at the posterior pole. Invest Ophthalmol Vis Sci 1996;37:1994-2001.

20 Shahidi M, Zeimer RC, Mori M. Topography of the retinal thickness in normal subjects. Ophthalmology 1990;97: $1120-4$.

21 Orgul S, Cioffi GA, Bacon DR, et al. Sources of variability of topometric data with a scanning laser ophthalmoscope. Arch Ophthalmol 1996;114:161-4.

22 Rohrschneider K, Burk ROW, Volcker HE, et al. Factors influencing three-dimensional data in follow-up studies with the laser tomographic scanner. In: Nasemann JE, Burk ROW, ed. Scanning laser ophthalmoscopy and tomography. Munich: Quintessenz Verlags, 1990;183-91.

23 Spencer AF, Sadiq SA, Pawson P, et al. Vertical optic disc diameter: discrepancy between planimetric and SLO measurements. Invest Ophthalmol Vis Sci 1995;36:796803.

24 Janknecht P, Funk J. Optic nerve head analyser and Heidelberg retina tomograph: accuracy and reproducibility of topographic measurements in a model eye and in volunteers. Br F Ophthalmol 1994;78:760-8. 